\title{
BMJ Open Research priorities and potential methodologies to inform care in subsequent pregnancies following stillbirth: a web-based survey of healthcare professionals, researchers and advocates
}

\author{
Aleena M Wojcieszek, ${ }^{\oplus 1}$ Alexander EP Heazell, ${ }^{\odot 2,3}$ Philippa Middleton, ${ }^{1,4}$
} David Ellwood, ${ }^{1,5}$ Robert M Silver, ${ }^{6}$ Vicki Flenady ${ }^{\oplus}$

To cite: Wojcieszek AM, Heazell AEP, Middleton P, et al. Research priorities and potential methodologies to inform care in subsequent pregnancies following stillbirth: a webbased survey of healthcare professionals, researchers and advocates. BMJ Open 2019;9:e028735. doi:10.1136/ bmjopen-2018-028735

- Prepublication history and additional material for this paper are available online. To view these files, please visit the journal online (http://dx.doi org/10.1136/bmjopen-2018028735).

Received 21 December 2018 Revised 3 April 2019 Accepted 4 June 2019

A Check for updates

(c) Author(s) (or their employer(s)) 2019. Re-use permitted under CC BY-NC. No commercial re-use. See rights and permissions. Published by BMJ.

For numbered affiliations see end of article.

\section{Correspondence to}

Aleena M Wojcieszek;

aleena.wojcieszek@mater.uq. edu.au

\section{ABSTRACT}

Objectives To identify research priorities and explore potential methodologies to inform care in subsequent pregnancies following a stillbirth.

Design Web-based survey by invitation.

Participants Multidisciplinary panel of 79 individuals involved in stillbirth research, clinical practice and/ or advocacy from the international stillbirth research community (response rate $=64 \%$ ).

Outcome measures Importance of 16 candidate research topics and perceived utility and appropriateness of randomised controlled trial (RCT) methodology for the evaluation of four pertinent interventions: (1) medical therapies for placental dysfunction (eg, antiplatelet agents); (2) additional antepartum fetal surveillance (eg, ultrasound scans); (3) early planned birth from 37 weeks gestation and (4) different forms of psychosocial support for parents and families.

Results Candidate research topics that were rated as 'important and urgent' by the greatest proportion of participants were: medical therapies for placental dysfunction ( $81 \%)$; additional antepartum fetal surveillance $(80 \%)$; the development of a core outcomes dataset for stillbirth research (79\%); targeted antenatal interventions for women who have risk factors $(79 \%)$ and calculating the risk of recurrent stillbirth according to specific causes of index stillbirth (79\%). Whether RCT methodologies were considered appropriate for the four selected interventions varied depending on the criterion being assessed. For example, while $72 \%$ of respondents felt that RCTs were 'the best way' to evaluate medical therapies for placental dysfunction, fewer respondents (63\%) deemed RCTs ethical in this context, and approximately only half (52\%) felt that such RCTs were feasible. There was considerably less support for RCT methodology for the evaluation of different forms of psychosocial support, which was reinforced by free-text comments.

Conclusions Five priority research topics to inform care in pregnancies after stillbirth were identified. There was support for RCTs in this area, but the panel remained divided on the
Strengths and limitations of this study

- This study offers a prioritisation of research topics to inform clinical practice for care in subsequent pregnancies following a stillbirth.

- A highly experienced sample of the international stillbirth research community was surveyed.

- It is the first study to directly explore professionals' perceptions regarding the appropriateness of randomised controlled trial methodology within the context of stillbirth research.

- Additional qualitative measures may have elicited a richer understanding of respondents' views on the more complex issues addressed.

- The views of parents and families are yet to be understood and should be explored in future research.

ethics and feasibility of such trials. Engagement with parents and families is a critical next step.

\section{INTRODUCTION}

Stillbirth has long-lasting medical and psychosocial consequences for women and families, and significant economic costs for parents and health services. ${ }^{1-4}$ Much of this emotional and economic burden relates to care in subsequent pregnancies. ${ }^{5-7}$ The majority of parents will conceive again after having a stillborn baby. ${ }^{8}$ These parents may face up to a fivefold increased risk of stillbirth in their next pregnancy, as well as increased risks of many other adverse pregnancy outcomes. ${ }^{9-12}$ The perceived risk of recurrent stillbirth is even higher than the absolute risk of $2.5 \%,{ }^{11}$ and many parents consequently experience profound anxiety and stress in their subsequent pregnancies. 561314 
Standard antenatal care is emotionally (and perhaps medically) unsuitable for many parents in pregnancies following a stillbirth. ${ }^{15}$ With this said, there is a lack of direct evidence as to what specific interventions or approaches to care might benefit these parents and their babies. ${ }^{16-18}$ Given the profound impacts of stillbirth and its potential consequences for subsequent pregnancies and far beyond, both families and care providers are likely to consider it unacceptable to do nothing above standard antenatal care in a pregnancy following stillbirth. The need for high-quality standards of care in this area has been reflected in the development of the recent Consensus Statement 'Management of pregnancy subsequent to stillbirth', endorsed by the Society of Obstetricians and Gynaecologists of Canada (SOGC). ${ }^{15}$

International data suggest that families and care providers are highly motivated to do as much as possible to maximise the health and well-being of parents and their unborn babies in pregnancies after perinatal death. Most parents, at least those in high-income regions, receive additional antenatal care visits and additional ultrasound scans (USS) in pregnancies following stillbirth. ${ }^{8121419}$ The option for elective early birth after 37 weeks of gestation appears common, particularly in pregnancies following late-gestation stillbirths (defined as $\geq 30$ weeks), compared with those following earlier gestation stillbirths. ${ }^{8}$ Elective caesarean section is also performed significantly more frequently in pregnancies following stillbirth, compared with pregnancies following live birth and pregnancies among primiparous women. ${ }^{19}$ Importantly, additional antepartum surveillance and elective early-term or late-term birth are primarily intended to detect and manage complications and/or mitigate medical risks, but are also generally welcomed by parents for psychological and social reasons. ${ }^{61419}$ But while these practices appear relatively commonplace in pregnancies following stillbirth, their benefits, risks and economic effects have yet to be evaluated formally among this population.

Medical therapies such as antiplatelet agents may also be used for women in pregnancies after stillbirth, particularly low-dose-aspirin (LDA). The use of LDA for these women is based largely on well-established evidence that LDA reduces placenta-mediated complications such as fetal growth restriction (FGR) and pre-eclampsia, ${ }^{20-24}$ which has been extrapolated to a probable protective effect against (recurrent) stillbirth. ${ }^{25}$ In a meta-analysis, LDA initiated at or before 16 weeks of gestation decreased the risk of perinatal death among women who had risk factors for pre-eclampsia. ${ }^{23}$ Heparin therapies such as low-molecular-weight heparin (LMWH) may also be prescribed to reduce the risk of stillbirth among women with previous placenta-mediated complications, although evidence to support this practice is limited, particularly in the absence of antiphospholipid antibodies. ${ }^{15}{ }^{26}$ Again, while these antiplatelet agents may theoretically reduce rates of recurrent stillbirth by attenuating the influence of certain pathological mechanisms, their efficacy has yet to be shown clearly in a randomised controlled trial (RCT).

A Cochrane Systematic Review on interventions to improve outcomes in subsequent pregnancies following stillbirth (defined as a fetal death of 20 weeks' gestation or more $)^{18}$ highlighted the challenges in gathering and synthesising evidence to inform clinical practice in this area. The review, including data from 10 RCTs and 222 women, assessed the effects of LDA, LMWH, immunotherapies and progestogen. No differences in recurrent stillbirth or other important outcomes were observed across the interventions assessed, but the analyses were not sufficiently powered to detect any such effects. In addition, the review was composed entirely of subsets of data which had been extracted from broader trials assessing interventions to reduce miscarriage together with stillbirth, which means that the trials were not designed to address recurrent stillbirth specifically. The quality of evidence in the review was judged to be low to very low, and no clear implications for clinical practice could be drawn due to insufficient evidence. ${ }^{18}$

While further data are needed to establish more targeted therapeutic and/or management strategies in the specific context of recurrent stillbirth, a number of potential barriers to generating such evidence arise. First, as stillbirth is statistically rare, very large sample sizes are required to achieve adequate power to assess (recurrent) stillbirth as a primary outcome, as emphasised in the aforementioned Cochrane Systematic Review. Second, a lack of clinical equipoise may preclude the evaluation of certain interventions via traditional RCT methodologies, as parents may decline to participate in trials where there is a chance that an intervention they believe may be beneficial will be withheld. Their treating clinicians may not endorse or facilitate such trials for the same reasons. Subtypes of RCT methodologies, such as cluster RCTs and stepped-wedge cluster RCTs, may provide a solution to some of these logistical and ethical barriers, ${ }^{27}{ }^{28}$ but these trials are complex, and they require careful planning, multicentre collaboration and substantial funding. Various criticisms and shortcomings of RCT methodology have also been cited alongside those mentioned here, and these apply across different areas of medicine. ${ }^{29-31}$

In addition to data informing clinical practice around antepartum surveillance, early scheduled birth, and medical therapies, other research gaps related to subsequent pregnancy care have also been identified, many of which were highlighted in the SOGC Consensus Statement. ${ }^{15}$ More precise estimates of stillbirth recurrence according to index causes of stillbirth may facilitate preconception counselling and aid in parents' decision-making around interventions in future pregnancies. ${ }^{25}{ }^{32}$ Until recently, ${ }^{33}$ little has been known about the effects of interpregnancy interval on subsequent pregnancy outcomes, and there remains an incomplete understanding of the broader psychological effects of interpregnancy interval on subsequent pregnancy experiences. ${ }^{8}$ Further, in relation to pregnancy subsequent to 
stillbirth, little is known about the longer term effects of early planned birth; the effects of additional antepartum fetal surveillance; preconception health interventions; peer support programmes; specialist clinical services for pregnancies after loss; how best to support siblings and partners during pregnancies after stillbirth; and optimal care provision in low- income and middle-income countries. ${ }^{8} 1825$

With finite research resources, it is important to establish which specific research areas have the most potential value to informing clinical practice, and are therefore most important and critical to pursue. It is also imperative to begin to formally explore the perceived value and acceptability of RCT methodologies for evaluating key interventions in this area. Therefore, the aim of this study was to consult an international, multidisciplinary panel of individuals involved in stillbirth research, clinical practice, and/or advocacy to determine research priorities and potential methodologies to inform clinical practice for care in pregnancies following a stillbirth.

\section{METHODS}

\section{Participants}

Participants comprised an international, multidisciplinary panel of stillbirth researchers, care providers and individuals involved in clinical practice, support and/or advocacy around stillbirth. One hundred and twenty-seven prospective participants were identified predominantly through their association with the International Stillbirth Alliance (ISA). The ISA (see: https://stillbirthalliance. $\mathrm{org} /$ ) is a not-for-profit international union of organisations and individual members invested in raising awareness of stillbirth, providing care and/or conducting research in all aspects of stillbirth prevention and care following stillbirth. Its annual scientific conference brings together academics, health professionals and parents to share the results of research and to promote and facilitate international research collaborations in the stillbirth field. In the present study, our aim was to capture the views of individuals involved in the ISA Scientific Network and relevant Working Groups, as well as individuals with specific experience and/or expertise in subsequent pregnancies following stillbirth. Therefore, participants in the present study were invited to take part on the basis of their involvement in or membership with one of more of the following:

- ISA Scientific Network (ie, nominated experts in scientific aspects of ISA work).

- ISA 'Risk and Prevention' or 'Clinical and Bereavement' Working Groups.

- The SOGC Consensus Statement: 'Management of pregnancy subsequent to stillbirth ${ }^{15}$ (initiated by invitation following the 2015 ISA annual scientific conference).

The authors of the present study are members of ISA and most were involved in the development of the aforementioned SOGC Consensus Statement, either as an author or member of its working group. ${ }^{15}$ Contact details for invitees were, therefore, drawn from the ISA database with the assistance of the ISA board, and/or were obtained through our existing professional connections.

\section{Measures}

A web-based survey was developed using Checkbox (Checkbox Survey, V.2017 Q2 SU2). The survey could be completed via computer, tablet or smartphone. The survey instrument measured background characteristics of participants; the perceived importance of a newly prepared list of 16 candidate research topics for informing clinical practice for care in pregnancies after stillbirth (described below); and the perceived utility and appropriateness of RCT methodology for four selected interventions relevant to pregnancy after stillbirth care (described below). The survey concluded with one optional open-ended item that provided an opportunity to add any general comments. The survey was piloted among the authorship team, a staff member of the coordinating centre of the Australian National Health and Medical Research Council Centre of Research Excellence in Stillbirth, and a volunteer from outside the field. Prior to its formal dissemination, modifications were made to the placement of questions and to the formatting of response options, to reduce the need for scrolling and to improve the survey's visual display and usability.

\section{Background characteristics of participants}

Geographical region of residence/work; duration of experience in stillbirth research/action; area of stillbirth research/action and area of clinical practice were assessed via categorical surveys items. Participants could select more than one area of stillbirth research/action if appropriate.

\section{Importance of candidate research topics}

Participants were presented with a prepared list of 16 candidate research topics in the context of pregnancies after stillbirth, which had been identified previously in studies and/or reviews. ${ }^{8151825}$ Candidate research topics covered a range of medical and psychosocial aspects of care for women and families, spanning preconception to labour (eg, 'Mode of birth and its associated medical and psychosocial outcomes for women and families', and 'The experiences and psychosocial outcomes of partners and siblings in pregnancies subsequent to stillbirth'), as well as epidemiological, economic and methodological topics (eg, 'Calculating the risk of recurrent stillbirth according to specific causes of index stillbirth', and 'The development of a core outcomes dataset for stillbirth (and recurrent stillbirth) research'). The full list of candidate research topics is shown in online supplementary file 1 .

The perceived importance of candidate research topics was assessed via a modified version of the GRADE working group approach to considering the relative importance of outcomes (http://www.gradeworkinggroup.org). Research topics were assessed on a nine-point rating scale where 1-3 = 'limited importance', 4-6 = 'important but 
not urgent and 7-9 = 'important and urgent'. An 'unsure' response option was also provided. Participants had the option to nominate a further two research topics not included in the prepared list, and could rate these the importance of these topics in the same manner described above. Participants were asked to consider the potential value to informing clinical practice when making all judgements.

\section{Utility and appropriateness of RCT methodologies}

The perceived utility and appropriateness of RCTs within the context of pregnancy after stillbirth research was assessed under four broad intervention categories: (1) medical therapies (eg, antiplatelet agents such as LDA and LMWH); (2) additional antepartum fetal surveillance (eg, USS); (3) early planned birth from 37 weeks' gestation and (4) different forms of psychosocial support for parents and families. Intervention categories 1-3 were selected based on their pertinence to modern clinical practice and common use in pregnancies after stillbirth, 8121419 and/or among women who have placenta-mediated pregnancy complications. ${ }^{20} 212324$ Category 4 (different forms of psychosocial support) was included on the basis of psychosocial support having been identified as a significant gap in research and practice in this area. $^{8141825}$

Within each category, the perceived utility and appropriateness of RCTs (umbrella term including traditional RCTs, cluster RCTs and stepped-wedge cluster RCTs) was assessed via five premises/statements:

- Best way: 'The best way to evaluate the effects of (intervention) for this population is to carry out RCTs.'

- Feasible: 'Depending on the specific methodology, it is feasible to carry out sufficiently powered RCTs of (intervention) for this population.'

- Ethical: 'Depending on the specific methodology, it is ethical to carry out RCTs of (intervention) for this population.'

- Improve implementation: 'Evidence from RCTs showing that (intervention) reduce/reduces the risk of recurrent stillbirth will improve implementation (ie, will result in higher uptake of the intervention).'

- Refine routine practice: 'Evidence from RCTs showing that (intervention) do not/does not reduce the risk of recurrent stillbirth will result in them/it being removed from routine clinical practice for this population.'

The wording of the final two statements was altered necessarily for the psychosocial intervention category (see online supplementary file 1). Agreement with the statements was measured on five-point Likert scales where (1) 'strongly disagree', (2) 'disagree', (3) 'neither agree nor disagree', (4) 'agree' and (5) 'strongly agree'. To provide context and aid in the meaningful interpretation of the data, the population referred to in the questions was women in high-income countries who have had a previous unexplained stillbirth or a stillbirth where placental dysfunction was implicated (eg, pre-eclampsia, FGR).

\section{Procedure}

Participants were invited to take part via direct email from the first author. The email contained brief information about the study and the survey link, which opened to a covering page explaining the study in more detail, alongside a button to commence the survey. The survey was designed to take 10-15 min to complete. Unless otherwise stated in the measures above, all items were mandatory and participants were required to select a response to each item before they could proceed to subsequent pages in the survey. Participation was voluntary and anonymous; participants were not asked for any identifying information as part of the survey instrument.

Survey reminders were sent at approximately 1, 2 and 6 weeks from the initial survey invitation. Participants were advised that the survey was closed to the invited participants only, and were asked not to circulate the survey link. On completion of the survey, participants were given the option to provide their name and professional affiliation/s in a separate online form, should they wish to be acknowledged as a member of the expert panel contributing to the study. The separate online form opened in a new website browser and the details provided via this form were not associated with participants' survey responses. Data were collected from 29 June to 31 August 2018.

\section{Analytic strategy}

Quantitative data were assessed using descriptive statistics expressed as frequencies and proportions, in aggregate form. The top five research topics were identified according to the proportions of panel members rating the research topic on points seven to nine of the scale (ie, corresponding to a scale label of 'important and urgent'). Analyses were performed using IBM SPSS Statistics V.22 (IBM). Responses to the open-ended item were assessed by two authors (AMW and VF). Comments were read independently, and summarised following discussion according to the principles of thematic analysis. ${ }^{34}$

\section{Patient and public involvement}

Patients and/or public were not expressly involved in the design or conduct of the current study.

\section{RESULTS}

Email invitations were disseminated on 29 June 2018. Three email invitations did not reach the recipients and, as alternative email addresses could not be sourced, these participants were removed from the denominator $(\mathrm{n}=124)$. Seventy-nine completed surveys were submitted (response rate $=64 \%$ ). Of these, one participant reported technical difficulties with the survey and completed a hard copy of the instrument, which was scanned and 
Table 1 Background characteristics of participants $(n=79)$

$\mathrm{n}=79$

Freq (\%)

\begin{tabular}{lc}
\hline Geographical region of residence/work & \\
Australia or New Zealand & $24(30.4)$ \\
Africa or Asia & $4(5.1)$ \\
Europe & $11(13.9)$ \\
North America & $24(30.4)$ \\
South or Central America & $3(3.8)$ \\
UK or Ireland & $13(16.5)$
\end{tabular}

Duration of experience in stillbirth research/action

\begin{tabular}{|c|c|}
\hline Less than 2 years & $6(7.6)$ \\
\hline 2-5years & $15(19)$ \\
\hline $6-9$ years & $16(20.3)$ \\
\hline 10-20years & $27(34.2)$ \\
\hline More than 20 years & $15(19)$ \\
\hline \multicolumn{2}{|l|}{ Area of stillbirth research/action* } \\
\hline Epidemiology/public health & $28(35.4)$ \\
\hline General practice/family medicine & $5(6.3)$ \\
\hline Midwifery & $18(22.8)$ \\
\hline Nursing (related to maternity care) & $4(5.1)$ \\
\hline Neonatology & $2(2.5)$ \\
\hline Obstetrics and/or gynaecology & $30(38)$ \\
\hline Pathology & $6(7.6)$ \\
\hline Psychology, psychiatry or social work & $13(16.5)$ \\
\hline Parent support and/or advocacy & $29(36.7)$ \\
\hline 'Other' & $4(5.1)$ \\
\hline \multicolumn{2}{|l|}{ Working in clinical practice } \\
\hline Yes & $50(63.3)$ \\
\hline No & $29(36.7)$ \\
\hline \multicolumn{2}{|l|}{ Area of clinical practice $(n=50)$} \\
\hline General practice/family medicine & $3(3.8)$ \\
\hline Midwifery & $13(16.5)$ \\
\hline Nursing (related to maternity care) & $2(2.5)$ \\
\hline Neonatology & $2(2.5)$ \\
\hline Obstetrics and/or gynaecology & $27(34.2)$ \\
\hline Pathology & $3(3.8)$ \\
\hline
\end{tabular}

*Participants could select more than one option.

Percentages may not equal 100 due to rounding.

emailed to the first author and entered into the survey system for collective analyses.

\section{Background characteristics of participants}

Table 1 presents the background characteristics of the panel. Participants most commonly resided in Australia and New Zealand (30\%) and North America (30\%). Seven participants $(9 \%)$ resided in Africa, Asia and South or Central America. Most participants (53\%) had been

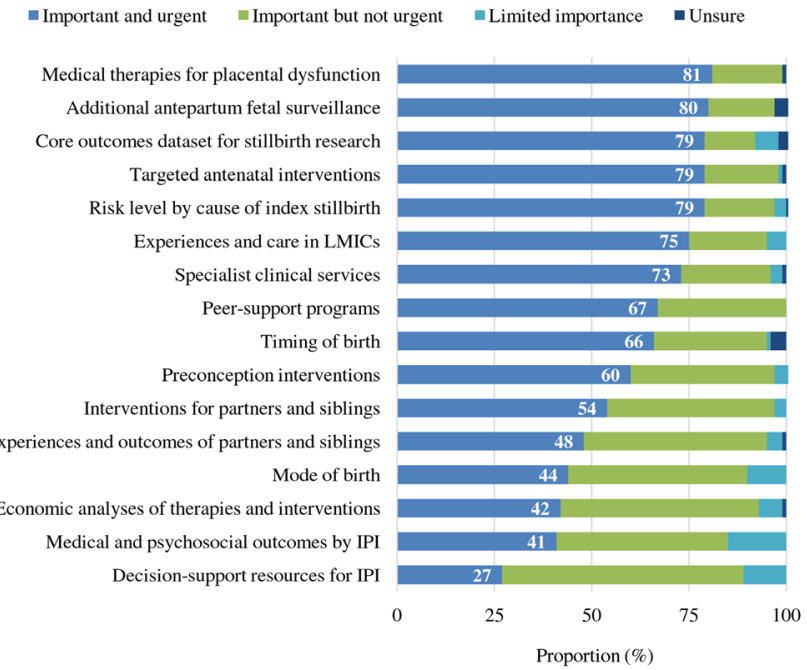

Figure 1 Importance of candidate research topics for informing care in pregnancies after stillbirth $(n=79)$. Items sorted in descending order according to the proportion of participants rating research topics as 'important and urgent' (7 through 9 on the 9-point rating scale); proportions of 'important and urgent' ratings marked inside bars. The detailed list of candidate research topics is shown in online supplementary file 1. IPI: interpregnancy interval; LMICs: low-income and middle-income countries.

involved in stillbirth research/action for 10 years or more, most commonly in the areas of parent support and/or advocacy (37\%), epidemiology/public health (35\%), and obstetrics and gynaecology (38\%). Most (63\%) were also working in clinical practice, most commonly in obstetrics and/or gynaecology (34\%) and midwifery (17\%).

\section{Importance of candidate research topics}

Responses regarding the importance of candidate research topics were negatively skewed, with the median responses ranging from six to eight on the nine-point scale (data not shown).

Figure 1 presents the distribution of responses according to the reference response categories. The proportion of participants who considered research topics to be 'important and urgent' ranged from $27 \%$ (decision-support resources for interpregnancy interval) to $81 \%$ (medical therapies for placental dysfunction). The proportion of participants who considered research topics to be 'important but not urgent' ranged from $13 \%$ (core outcomes dataset for stillbirth research) to $62 \%$ (decision-support resources for interpregnancy interval). Only three topics were thought to have "limited importance' by $10 \%$ or more of participants. These three topics were: medical and psychosocial outcomes according to interpregnancy interval, decision-support resources for interpregnancy interval and mode of birth and its associated medical and psychosocial outcomes (see figure 1).

The top five research topics, which were assessed as 'important and urgent' by more than three-quarters of participants, are presented in the box 1 . 
Box 1 Top five research topics for care in pregnancies after stillbirth

1. Medical therapies for placental dysfunction (eg, antiplatelet agents such as low-dose-aspirin) and their associated medical and psychosocial outcomes for women and families.

2. Additional antepartum fetal surveillance (eg, additional ultrasound scan) and its associated medical and psychosocial outcomes for women and families.

3. The development of a core outcomes dataset for stillbirth (and recurrent stillbirth) research.

4. Targeted antenatal interventions for women with risk factors (eg, diabetes, smoking, alcohol use) and their associated medical and psychosocial outcomes for women and families.

5. Calculating the risk of recurrent stillbirth according to specific causes of index stillbirth.

Thirty-eight additional research topics were suggested by 28 participants. Common themes in the topics suggested included examination of the causes of stillbirth $(\mathrm{n}=7)$; education and support for healthcare providers $(\mathrm{n}=7)$; further suggestions regarding the psychosocial care of women and families $(n=6)$; and care and resources for specific populations of women (eg, non-English speaking women) $(\mathrm{n}=4)$. Some participants used this section of the survey to reiterate the importance of topics presented in the prepared list (eg, 'More research is needed in low-middle-income countries where $98 \%$ of stillbirths occur-across all areas including support for women after stillbirth, etc'). Where rated, the importance of these additional research topics ranged from six to nine, with most being considered 'important and urgent'.

\section{Utility and appropriateness of RCT methodologies}

Figure 2 presents the proportion of participants who agreed or strongly agreed with the given statements within each intervention category. Agreement that RCTs were the 'best way' to evaluate the given interventions ranged from $39 \%$ (different forms of psychosocial support) to $72 \%$

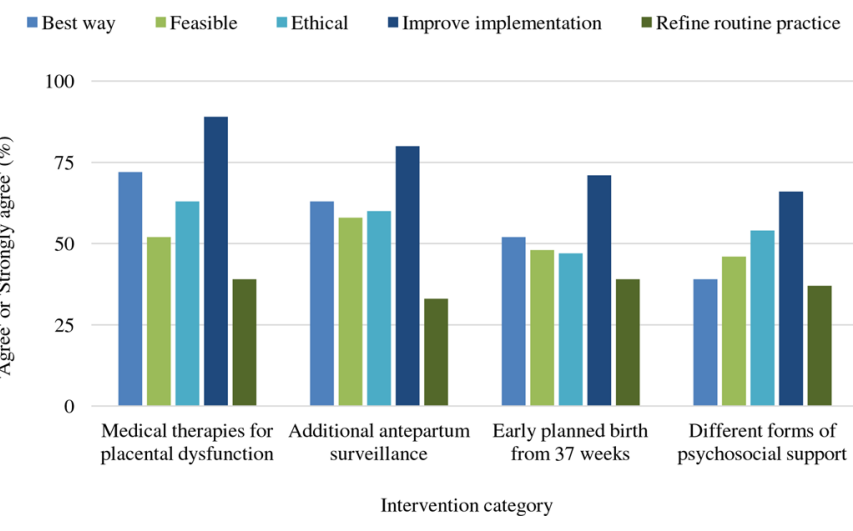

Figure 2 Utility and appropriateness for RCT methodologies for the evaluation of four selected intervention categories according to the five criteria $(n=79)$. The detailed description of intervention categories and assessment criteria are shown in online supplementary file 1. RCT: randomised controlled trial. (medical therapies for placental dysfunction). Approximately half of participants $(46 \%-52 \%)$ agreed that RCTs were logistically feasible across the interventions and slightly more (47\%-63\%) agreed that RCTs were ethically sound across the interventions. Most participants agreed that evidence from RCTs showing a reduction in the risk of recurrent stillbirth would improve implementation of the interventions, ranging from $66 \%$ for different forms of psychosocial support to $89 \%$ for medical therapies for placental dysfunction. A much smaller proportion (33\%-39\%) agreed that evidence from RCTs, showing the intervention did not reduce the risk of recurrent stillbirth (or that the intervention was unhelpful or least beneficial in the case of psychosocial support), would help to refine routine clinical practice. In fact, up to $43 \%$ of participants disagreed or strongly disagreed with this premise (see online supplementary file 1 for raw data on this outcome).

\section{Additional comments from respondents}

Twenty-four participants $(30 \%)$ provided comment before concluding this survey. The comments provided were relatively brief; the complete dataset included approximately 1200 words. A number of participants echoed the view that, while RCT methodologies were valuable, significant problems arise in the adoption of RCTs within the stillbirth research context. These problems included logical difficulties in obtaining (or even forecasting) the requisite sample sizes, the potential for contamination of interventions, and ethical concerns about clinical equipoise and RCT methodologies being incompatible with a much-needed individualised approach to care.

While I support the concept of RCT...the numbers may be overwhelming... (Response ID: 1694866)

It is clear from the AFFIRM trial, and others like it, that even if you THINK you are adequately powered for stillbirth that you are probably not...AND (in our information rich world) that people in both arms of an 'intervention' study can get access to the 'intervention' enough to significantly dilute any effect you hope to see in a rare outcome like stillbirth (and/ or subsequent pregnancy) (Response ID: 1696087) ${ }^{35}$

It would appear that families have very different responses and needs after a stillbirth, which is why I don't think RCTs would be appropriate to evaluate most interventions. (Response ID: 1696046).

Some participants believed it would be especially problematic to adopt RCT methodologies to evaluate different psychosocial interventions (against one another). As one participant stated:

...It is particularly concerning that you would even ask if a RCT is suitable to test psychosocial support as this would NEVER be a suitable approach whatever the 'intervention'. (Response ID: 1696087) 
Other participants referred to the evidence-practice gap, noting that even high-quality RCT evidence does not guarantee the implementation of interventions into clinical practice. As expressed by one respondent, a conflict may arise between the needs of individual families and what may be outlined in relevant clinical practice guidance.

I think that the there [sic] will be continual tension between medical evidence (ie, take this pill or have this ultrasound) and what families want/prefer in a pregnancy after stillbirth. This is especially true when families feel medical tests let them down before (ie, they had a 'normal' ultrasound the day before their stillbirth. I think it will be important to always include the voices of families in research, and especially in clinical 'protocols' that come from the evidence. How do we as care providers best respond when what families want in subsequent pregnancies isn't what is evidence-based (ie, routine NSTs, frequent ultrasounds, etc.)? (Response ID: 1691817)

The need to consider the potential for iatrogenic adverse outcomes following early scheduled birth was also raised by one respondent:

I missed the fact that by intervention early (delivery 37 weeks) adverse outcomes are seen in the neonate. Recurrent stillbirth is disastrous but still rare. Other adverse outcomes should be taken into account in your risk management... (Response ID: 1698731)

The remaining comments varied, but included additional suggestions for future research; expressions of the importance of the topic and of the present study; and that high-quality psychosocial support is imperative during pregnancies after loss, given the profound anxiety many parents and care providers experience during this time. The need to engage affected parents in the development of clinical protocols and interventions was also articulated clearly. Importantly, none of the 24 respondents to this item expressed explicit and unqualified support for RCTs in their comments, though one participant felt that animal studies of treatments for placental dysfunction would be valuable and could potentially justify (or rule-out) RCTs in this specific area.

\section{DISCUSSION}

\section{Main findings}

This study has identified five priority research topics to inform clinical practice for the care of families in pregnancies following a stillbirth. Views regarding the utility and appropriateness of RCT methodology (including traditional RCTs, cluster RCTs and stepped-wedge cluster RCTs), in the evaluation of four pertinent interventions were also explored. In crude terms, the quantitative data demonstrated more support than not for such RCTs, but this depended on the nature of the intervention and the criterion being assessed. RCT methodology was clearly considered more acceptable for the evaluation of medical therapies for placental dysfunction and of additional antepartum surveillance, than it was for the evaluation of early planned birth (from 37 weeks' gestation) and different forms of psychosocial support for parents and families. While the quantitative data indicated some level of endorsement of RCTs, optional free-text comments provided at the conclusion of the survey painted a different picture. These comments noted broad concerns about RCT methodology in this context, particularly with regard to the evaluation of different forms of psychosocial support. It is possible that respondents who felt steadfast opposition towards RCT methodology (regardless of the intervention) were more likely to complete this item of the survey and express their views than respondents who saw RCTs as less problematic. Nonetheless, overall, support for RCT methodology was far from overwhelming among this experienced sample of the international stillbirth research community, which may have important implications for the facilitation of RCTs in this area.

\section{Interpretation of findings}

The candidate research topics emerging as most pressing were broadly in line with the major research gaps identified in the Cochrane Systematic Review on this topic. ${ }^{18}$ As a first priority, medical therapies for placental dysfunction appear to warrant research investment. The evaluation of additional antepartum surveillance was also highly supported, consistent with a recent review. ${ }^{25}$ Most parents in high-income settings receive additional antenatal care visits and additional USS in pregnancies after stillbirth, ${ }^{8121419}$ and thus the panel appeared to have acknowledged a need to better understand the effects of these practices. Development of a core outcomes dataset was also prioritised, and it is encouraging to see such work underway. ${ }^{36}$ It is somewhat surprising that timing of birth and its associated medical and psychosocial outcomes was rated relatively low on the scale of importance, given the inclination towards early scheduled birth in pregnancies after stillbirth and its considerable risk of iatrogenic adverse outcomes. ${ }^{37}$ It is possible that there were greater ethical and logistical concerns around research on this particular question, as evidenced by respondents' views regarding the appropriateness of RCTs in the evaluation of early planned birth.

Of the four interventions explored, medical therapies for placental dysfunction appeared to be perceived as most suitable for evaluation via RCT methodology. It may be that LDA, due to its simplicity and low financial cost, lends itself well to evaluation via such clinical trials. The relative ease of measurement and reduced risk of contamination of interventions may also place LDA in a favourable position when compared with more complex interventions. In the present study, a significant majority (71\%) believed RCTs were the 'best way' to evaluate such medical therapies. Participants, therefore, appeared to consider the major obstacle for RCTs of this intervention to be the feasibility of obtaining the requisite sample 
sizes, which is likely only achievable through adopting a pragmatic, multicentre design, such as that in the recent AFFIRM trial ${ }^{38}$ and ongoing My Baby's Movements trial (ACTRN12614000291684). Such trials would need to contend with potential challenges to recruitment, given issues around clinical equipoise, and given LDA's low financial cost and safety of short-term use ${ }^{39}$ (the same factors that lend LDA well to clinical trial evaluation). The prospect of a placebo-controlled parallel RCT of LDA is uncertain; this would depend on the setting(s) and would require extensive formative research with and among parents. Parallel trials may reasonably be employed to assess LDA against combined LDA and LMWH, or to compare different doses of LDA, for instance.

For the three medical intervention categories explored, the majority of participants felt that RCT evidence showing a reduction in the risk of recurrent stillbirth would enhance the implementation of those interventions. However, far fewer (33\%-39\%) felt that the opposite would be true; that is, that RCT evidence showing that the interventions did not reduce the risk of recurrent stillbirth, would prompt the cessation of these interventions within routine clinical practice. As these interventions are not necessarily performed solely to reduce to chance of recurrent stillbirth, this finding is not entirely unexpected. Such interventions may be carried out with an intention to reduce the risk of other adverse outcomes, as well as due to important psychosocial factors, at least in regard to additional antepartum surveillance and early scheduled birth. Empathy and the imperative to do something in the face of such a distressing situation is likely to be a highly influential factor in clinician behaviour, particularly if parents explicitly request such an intervention. Indeed, this 'tension' between medical evidence and the needs and preferences of families was well articulated by participants in the current study. Nonetheless, it is possible that the findings also speak, at least partially, to the difficulty and complexity of 'deimplementation' of embedded clinical practices and 'unlearning' of established clinical knowledge and assumptions. ${ }^{40-42}$

Importantly, for any medical intervention, the index cause of stillbirth will influence the appropriateness of that intervention and its likelihood of effectiveness. ${ }^{18} 43$ The index cause of stillbirth and its associated recurrence risk may also influence parents' willingness to participate in clinical trials of certain medical interventions, especially depending on the design of those trials. These premises reinforce the need for more research on the risk of recurrent stillbirth according to specific index causes of death, which emerged as a priority in the present study. More comprehensive understanding of parents' perceptions of risk in pregnancies after stillbirth would also be highly informative.

\section{Strengths and limitations}

The present study is the first to prioritise research topics in the area of subsequent pregnancies following a stillbirth, and it is the first to directly explore perceptions regarding the appropriateness of RCT methodology to evaluate certain interventions in this context. Its strengths include a respectable survey response rate, resulting in a sample of highly experienced participants, with more than half being involved in stillbirth research/action for 10 years or more. A limitation of this study was the restriction of the sample largely to professionals. While $37 \%$ of participants aligned themselves with parent support and/or advocacy, parents and families were not targeted directly via our recruitment strategy and the findings, therefore, cannot be generalised to the broader community of individuals and communities affected by stillbirth. We acknowledge that not all individuals conducting research or working in this area are necessarily affiliated with ISA or participated in the SOGC Consensus Statement, and therefore that the views of any such individuals have not have been captured in the present study. Additionally, due to the nature and content of the survey, and its response base, the research directions most relevant to low-income and middle-income countries have not been addressed. It should also be noted that the appropriateness of RCT methodology in stillbirth research is a particularly complex issue. Quantitative measurement of perceptions on this issue via web-based survey is somewhat cursory, although the free-text comments provided at the conclusion of the survey appeared to capture many of the important nuances at play.

\section{Future research}

Research to understand the views of parents and families is a critical next step. A priority setting exercise which involved over 574 parents already identified support for women and their partners in subsequent pregnancies as a research priority. ${ }^{44}$ Therefore, future research should also explore families' views regarding (broad) methodologies to carry out such research. Carefully designed, pragmatic RCTs have an important role and are not out of the question, but it is unrealistic to expect that we can rely solely on the evidence of RCTs in the formulation of clinical practice guidance in this area, as well as many other areas of medicine. ${ }^{45}$ Certainly, the engagement of parents and families in the development and evaluation of any interventions related to pregnancy after a stillbirth is of paramount importance, regardless of the methodology. It is also vital to consider psychosocial support alongside the evaluation of any interventions that have a predominantly medical focus.

\section{Author affiliations}

${ }^{1}$ NHMRC Centre of Research Excellence in Stillbirth, Mater Research Institute - The University of Queensland (MRI-UQ), South Brisbane, Queensland, Australia

${ }^{2}$ Maternal and Fetal Health Research Centre, University of Manchester, Manchester, UK

${ }^{3}$ St Mary's Hospital, Manchester University NHS Foundation Trust, Manchester Academic Health Science Centre, Manchester, UK

${ }^{4}$ South Australian Health and Medical Research Institute (SAHMRI), Adelaide, South Australia, Australia

${ }^{5}$ School of Medicine, Griffith University and Gold Coast University Hospital, Gold Coast, Queensland, Australia 
${ }^{6}$ Department of Obstetrics and Gynecology, University of Utah, Salt Lake City, Utah, USA

Acknowledgements We thank the expert panel contributing to the study for their time and insights (in alphabetical order; list displays the names of the individuals who chose to be listed in the expert panel by completing the associated (separate) online form: Abdul-Razak S Abdul-Mumin; Helen D Bailey; Jon F R Barrett; Hannah Blencowe; Frances M Boyle; Jillian Cassidy; Paul R Cassidy; Jane Dahlstrom; Guilherme Ramires de Jesus; Paula Dillon; Wes Duke; Christine East; Jan Jaap H M Erwich; Claire Everard; Lynn L Farrales; Ms Megan E Fockler; Ruth C Fretts; Glenn Gardener; Karen J Gibbins; Sanne J Gordijn; Susan Heath; Jacqui Hylton; Nasrin Javid; Belinda Jennings; T Yee Khong; Nisha Khot; Fleurisca J Korteweg; Noor Niyar N Ladhani; Tina Lavin; Susannah Hopkins Leisher; Shauna Libsack; Elizabeth McClure; Lesley McCowan; Sarah Meaney; Dana Muin; Margaret M Murphy; Dang Thi Nghia; Daniel Nuzum; Jeremy J N Oats; Joann O'Leary; Robert Pattinson; Veronica Pingray; Claudia Ravaldi; Hanna Reinebrant; Jessica Ruidiaz; Caroline H Schmeer; Louise Stephens; Claire Storey; Alfredo Vannacci; R Douglas Wilson; Lindsey J Wimmer and LeighAnne Wright. We thank Katy Gold and Jillian Cassidy for assisting to compile the list of ISA invitees. We also thank Sarah Henry for her assistance with obtaining the research authorisations required to carry out this study, and Janine Rix and Sarah Henry for piloting the survey and providing feedback to enhance its design and usability.

Contributors AMW: contributed to study conception and developed the survey instrument, managed data collection, conducted quantitative analyses, cosummarised free-text responses, interpreted the findings, drafted and edited the manuscript. AEPH and RMS: contributed to the development of the survey instrument, interpretation of findings, review and editing. PM and DE: contributed to study conception, development of the survey instrument, interpretation of findings, review and editing. VF: contributed to study conception, development of the survey instrument, interpretation of findings, review and editing and cosummarised the free-text responses. All authors reviewed the final version of the manuscript.

Funding The authors have not declared a specific grant for this research from any funding agency in the public, commercial or not-for-profit sectors.

Competing interests AMW is an associate investigator on the Australian NHMRC Centre of Research Excellence in Stillbirth. PM, DE and VF are principal investigators on this same grant and VF and DE direct and codirect this centre, respectively. AEPH is the clinical lead for a specialist clinical service for women who have experienced a stillbirth or perinatal death. He has no financial conflict of interest to declare. DE has received sitting fees from the Australian Medical Council, but this work is not related to the current study. DE has received payment for providing expert witness reviews for medicolegal cases unrelated to this study. RMS has been awarded NIH grants unrelated to this work. He has carried out paid consultancy for Gestavision (a company developing a diagnostic for pre-eclampsia) and has received payment for grand rounds at several universities. All authors are members of the ISA Scientific Network.

Ethics approval This study was approved by the Mater Health Services Human Research Ethics Committee on 22 June 2018 (Reference No: HREC/18/MHS/85), within the guidelines of the Australian National Statement on Ethical Conduct in Human Research (2007) updated 2015.

Provenance and peer review Not commissioned; externally peer reviewed.

Data sharing statement Raw outcome data not included in the present article are available on reasonable request.

Open access This is an open access article distributed in accordance with the Creative Commons Attribution Non Commercial (CC BY-NC 4.0) license, which permits others to distribute, remix, adapt, build upon this work non-commercially, and license their derivative works on different terms, provided the original work is properly cited, appropriate credit is given, any changes made indicated, and the use is non-commercial. See: http://creativecommons.org/licenses/by-nc/4.0/.

\section{REFERENCES}

1. Burden C, Bradley S, Storey C, et al. From grief, guilt pain and stigma to hope and pride - a systematic review and meta-analysis of mixed-method research of the psychosocial impact of stillbirth. BMC Pregnancy Childbirth 2016;16:9.

2. Flenady V, Wojcieszek AM, Middleton P, et al. Stillbirths: recall to action in high-income countries. The Lancet 2016;387:691-702.

3. Heazell AEP, Siassakos D, Blencowe $\mathrm{H}$, et al. Stillbirths: economic and psychosocial consequences. The Lancet 2016;387:604-16.
4. Ogwulu CB, Jackson LJ, Heazell AE, et al. Exploring the intangible economic costs of stillbirth. BMC Pregnancy Childbirth 2015;15:188.

5. Meaney S, Everard CM, Gallagher S, et al. Parents' concerns about future pregnancy after stillbirth: a qualitative study. Health Expect 2017;20:555-62.

6. Mills TA, Ricklesford C, Cooke A, et al. Parents' experiences and expectations of care in pregnancy after stillbirth or neonatal death: a metasynthesis. BJOG 2014;121:943-50.

7. Mistry $\mathrm{H}$, Heazell $A E$, Vincent $\mathrm{O}$, et al. A structured review and exploration of the healthcare costs associated with stillbirth and a subsequent pregnancy in England and Wales. BMC Pregnancy Childbirth 2013:13:236.

8. Wojcieszek AM, Boyle FM, Belizán JM, et al. Care in subsequent pregnancies following stillbirth: an international survey of parents. BJOG 2018;125:193-201.

9. Getahun D, Lawrence JM, Fassett MJ, et al. The association between stillbirth in the first pregnancy and subsequent adverse perinatal outcomes. Am J Obstet Gynecol 2009;201-378.e1-378.e6.

10. Heinonen S, Kirkinen P. Pregnancy outcome after previous stillbirth resulting from causes other than maternal conditions and fetal abnormalities. Birth 2000;27:33-7.

11. Lamont K, Scott NW, Jones GT, et al. Risk of recurrent stillbirth: systematic review and meta-analysis. BMJ 2015;350:h3080.

12. Monari F, Pedrielli G, Vergani P, et al. Adverse perinatal outcome in subsequent pregnancy after stillbirth by placental vascular disorders. PLoS One 2016;11:e0155761.

13. Gravensteen IK, Jacobsen EM, Sandset PM, et al. Anxiety, depression and relationship satisfaction in the pregnancy following stillbirth and after the birth of a live-born baby: a prospective study. BMC Pregnancy Childbirth 2018;18:41.

14. Mills TA, Ricklesford C, Heazell AEP, et al. Marvellous to mediocre: findings of national survey of UK practice and provision of care in pregnancies after stillbirth or neonatal death. BMC Pregnancy Childbirth 2016;16:1-10.

15. Ladhani NNN, Fockler ME, Stephens L, et al. No. 369-Management of pregnancy subsequent to stillbirth. J Obstet Gynaecol Can 2018;40:1669-83.

16. Monari F, Facchinetti F. Management of subsequent pregnancy after antepartum stillbirth. A review. J Matern Fetal Neonatal Med 2010;23:1073-84.

17. Reddy UM. Prediction and prevention of recurrent stillbirth. Obstet Gynecol 2007;110:1151-64.

18. Wojcieszek AM, Shepherd E, Middleton $\mathrm{P}$, et al. Care prior to and during subsequent pregnancies following stillbirth for improving outcomes. Cochrane Database Syst Rev 2018;12:CD012203.

19. Gravensteen IK, Jacobsen E-M, Sandset PM, et al. Healthcare utilisation, induced labour and caesarean section in the pregnancy after stillbirth: a prospective study. BJOG: An International Journal of Obstetrics \& Gynaecology 2018;125:202-10.

20. Askie LM, Duley L, Henderson-Smart DJ, et al. Antiplatelet agents for prevention of pre-eclampsia: a meta-analysis of individual patient data. The Lancet 2007;369:1791-8.

21. Bujold E, Roberge S, Lacasse Y, et al. Prevention of preeclampsia and intrauterine growth restriction with aspirin started in early pregnancy: a meta-analysis. Obstet Gynecol 2010;116:402-14

22. Duley L, Henderson-Smart DJ, Meher S, et al. Antiplatelet agents for preventing pre-eclampsia and its complications. Cochrane Database Syst Rev 2007;CD004659.

23. Roberge S, Nicolaides KH, Demers S, et al. Prevention of perinatal death and adverse perinatal outcome using low-dose aspirin: a meta-analysis. Ultrasound Obstet Gynecol 2013;41:491-9.

24. Roberge S, Odibo AO, Bujold E. Aspirin for the prevention of preeclampsia and intrauterine growth restriction. Clin Lab Med 2016;36:319-29.

25. Fockler ME, Ladhani NNN, Watson J, et al. Pregnancy subsequent to stillbirth: Medical and psychosocial aspects of care. Semin Fetal Neonatal Med 2017;22:186-92.

26. Duffett L, Rodger M. LMWH to prevent placenta-mediated pregnancy complications: an update. Br J Haematol 2015;168:619-38.

27. Hussey MA, Hughes JP. Design and analysis of stepped wedge cluster randomized trials. Contemp Clin Trials 2007;28:182-91.

28. Mdege ND, Man M-S, Taylor (nee Brown) CA, et al. Systematic review of stepped wedge cluster randomized trials shows that design is particularly used to evaluate interventions during routine implementation. J Clin Epidemiol 2011;64:936-48.

29. Bothwell LE, Greene JA, Podolsky SH, et al. Assessing the gold standard-lessons from the history of RCTs. $N$ Engl J Med 2016;374:2175-81.

30. Carey TA, Stiles WB. Some problems with randomized controlled trials and some viable alternatives. Clin Psychol Psychother 2016;23:87-95. 
31. Mulder R, Singh AB, Hamilton A, et al. The limitations of using randomised controlled trials as a basis for developing treatment guidelines. Evid Based Ment Health 2018;21:4-6.

32. Wojcieszek AM, Shepherd E, Middleton P, et al. Care prior to and during subsequent pregnancies following stillbirth for improving outcomes. Cochrane Database Syst Rev 2016;CD012203.

33. Regan AK, Gissler M, Magnus MC, et al. Association between interpregnancy interval and adverse birth outcomes in women with a previous stillbirth: an international cohort study. The Lancet 2019;393:1527-35.

34. Braun V, Clarke V. Using thematic analysis in psychology. Qual Res Psychol 2006;3:77-101.

35. Norman JE, Heazell AEP, Rodriguez A, et al. AFFIRM investigators. Awareness of fetal movements and care package to reduce fetal mortality (AFFIRM): a stepped wedge, cluster-randomised trial. The Lancet 2018;392:1629-38.

36. Core Outcome Measures in Effectiveness Trials (COMET) initiative. COSTIL study: core outcomes in stillbirth trials. (cited 14 Nov 2018). Available from: http://www.comet-initiative.org/studies/details/982.

37. Robson S, Thompson J, Ellwood D. Obstetric management of the next pregnancy after an unexplained stillbirth: an anonymous postal survey of Australian obstetricians. Aust N Z J Obstet Gynaecol 2006;46:278-81.

38. Norman JE, Heazell AEP, Rodriguez A, et al. Awareness of fetal movements and care package to reduce fetal mortality
(AFFIRM): a stepped wedge, cluster-randomised trial. The Lancet 2018;392:1629-38.

39. Henderson JT, Whitlock EP, O'Connor E, et al. Low-dose aspirin for prevention of morbidity and mortality from preeclampsia: a systematic evidence review for the U.S. Preventive Services Task Force. Ann Intern Med 2014;160:695-703.

40. Cegarra-Navarro JG, Wensley AKP, Polo MTS. A conceptual framework for unlearning in a homecare setting. Knowledge Management Research \& Practice 2014;12:375-86.

41. Gupta DM, Boland RJ, Aron DC. The physician's experience of changing clinical practice: a struggle to unlearn. Implement Sci 2017;12:28.

42. Helfrich CD, Rose AJ, Hartmann CW, et al. How the dual process model of human cognition can inform efforts to de-implement ineffective and harmful clinical practices: A preliminary model of unlearning and substitution. J Eval Clin Pract 2018;24:198-205.

43. Wojcieszek AM, Shepherd E, Middleton P, et al. Interventions for investigating and identifying the causes of stillbirth. Cochrane Database Syst Rev 2018;CD012504.

44. Heazell AE, Whitworth MK, Whitcombe J, et al. Research priorities for stillbirth: process overview and results from UK Stillbirth Priority Setting Partnership. Ultrasound Obstet Gynecol 2015;46:641-7.

45. Faraoni D, Schaefer ST. Randomized controlled trials vs. observational studies: why not just live together? BMC Anesthesiol 2016;16:102. 